\title{
Anatomy of leaf abscission in the Amur honeysuckle (Lonicera maackii, Caprifoliaceae): a scanning electron microscopy study
}

\author{
Hua-Feng Wang • Cynthia M. Ross Friedman • \\ Jin-Chao Shi • Zhi-Yong Zheng
}

Received: 4 February 2010 /Accepted: 30 April 2010 /Published online: 29 May 2010

(C) Springer-Verlag 2010

\begin{abstract}
Lonicera maackii (Rupr.) Maxim. (Amur honeysuckle) is native to Asia and an important ornamental in China. However, the anatomy of leaf abscission (shedding) in L. maackii had not been studied previously. Such work is needed not only because knowledge of the leaf abscission process is important for a horticultural species like L. maackii but also because leaf abscission is probably the least understood abscission process, as it occurs so rapidly. Therefore, our objective was to use scanning electron microscopy (SEM) to examine the progression of leaf abscission in L. maackii at the cellular level. L. maackii branches with leaves were regularly collected in Beijing, China over the 2-month period in which leaves abscise, and examined with SEM. We found that, unlike in model species, the cortex is involved in abscission, forming an "abaxial gap." We discovered that there is no discrete abscission zone prior to the onset of abscission and that no cell divisions precede abscission. An abscission zone did become evident well after the abscission process had begun,
\end{abstract}

Corresponding authors:

C. Ross Friedman - English

J.-C. Shi - Chinese

\section{H.-F. Wang}

Beijing Urban Ecosystem Research Station, State Key Laboratory of Urban and Regional Ecology, Research Center

for Eco-Environmental Sciences, Chinese Academy of Sciences, Beijing 100085, China

\section{M. Ross Friedman $(\square)$}

Department of Biological Sciences, Thompson Rivers University, P. O. Box 3010, 900 McGill Road,

Kamloops, BC V2C 5N3, Canada

e-mail: cross@tru.ca

J.-C. Shi $(\bowtie) \cdot$ Z.-Y. Zheng

Horticultural Department, Beijing Vocational College of Agriculture, Beijing 102442, China

e-mail: shijinchao888@sina.com but its cells were enlarged, not constricted as in typical abscission zones. In the abaxial gap, intact cells separated at their middle lamella, but in the abscission zone, cell separation involved the entire wall, which is not typical. We did observe expected mechanical fission of vascular tissues. While the leaf abscission process we observed in L. maackii has similarities with model systems, aspects deviate from the expected.

Keywords Abaxial gap · Amur honeysuckle · Anatomy · Leaf abscission · Lonicera maackii . Scanning electron microscopy (SEM)

\section{Introduction}

Lonicera maackii (Rupr.) Maxim. (Amur honeysuckle) is a deciduous shrub belonging to the family Caprifoliaceae and is native to temperate Asia in northern and western China (south to Yunnan), Mongolia, Japan, Korea, and southeastern Russia (Xu and Wang 1988). In China, L. maackii is an ornamental plant and street tree, widely planted because of its fragrant flowers, long flowering period, and attractive red fruits.

However, while L. maackii is an important horticultural species, there have been no reports that describe the process of leaf abscission in the species. Abscission is the natural process by which organs such as leaves, fruits, buds, and flowers separate from the parent plant when such organs are diseased, stressed, or at the terminal stage of their development (González-Carranza et al. 1998). The abscission zone is defined as the region at the base of abscising organs through which abscission eventually occurs (Gawadi and Avery 1950). In the abscission zone of some species, but not all, cell division may produce several tiers of cells called the abscission layer. Anatomy of abscission is important in understanding the biology of a given plant species since form and structure comprise an appropriate 
starting point for potential functional comparisons between botanically distinct organs (van Nocker 2009). In horticulture, abscission plays an important role in determining the population of flowers and fruits and in the timing and effectiveness of harvest procedures. Thus, an understanding of abscission is important to the cultivation of a valuable ornamental species such as L. maackii.

The anatomy and physiology of abscission has been the subject of extensive research in several species. Sexton (1976) studied the development of the leaf abscission zone in Impatiens sultani Hook. with scanning and transmission electron microscopes, describing the nature and extent of the hydrolytic processes that lead to the separation of cells in the abscission zone. Mackenzie (1979) studied the structure of the fruit of the red raspberry (Rubus idaeus L.) in relation to abscission and revealed that two modes of cell separation occur within raspberry abscission zones. Sexton and Redshaw (1981) observed the morphological development process in the abscission of I. sultani leaves and discussed the significance of these observations relative to the general anatomy and mechanism of fracture of abscission zones. Roberts et al. (1984) observed ethylenepromoted tomato flower (Lycopersicon esculentum Mill.) abscission: exposure of excised pedicels to ethylene resulted in abscission accelerated by flower removal. It is apparent from the literature, however, that relatively few studies have been undertaken specifically on leaf abscission, likely because leaf structures can be shed quite rapidly: The sequence of events leading to separation are condensed into a short time frame and challenging to observe in their entirety.

In this paper, we report the results of our focused scanning electron microscopy (SEM) investigation of the anatomy of leaf abscission in L. maackii.

\section{Materials and methods}

We examined L. maackii growing at the Beijing Vocational College of Agriculture and the Beijing Botanical Garden in China. In order to observe the growth habit of these plants, whole shrubs were photographed with an Olympus SP-565UZ digital camera (Tokyo, Japan) during the summer growing season (from 1 April 2009 to 30 October 2009).

During the period when leaves of L. maackii abscise (1 November to 31 December 2009), branches were collected every 3 to 4 days (two branches per date) and photographed using a Nikon DXM 1200 stereo dissecting microscope (Tokyo, Japan). In preparation for SEM, we fixed the samples in FAA $550 \%$ ethanol, $5 \%(v / v)$ acetic acid, and $3.7 \%(v / v)$ formaldehyde] for at least $24 \mathrm{~h}$ and dehydrated through a tertiary butyl alcohol series (Jensen 1962). From the center of branches collected at different developmental stages, two leaves were cut transversely, and two were cut longitudinally in 95\% ethanol under the Nikon DXM 1200 stereo dissecting microscope, transferred through an ethanol iso-amyl acetate series (95\% ethanol, 100\% ethanol, $75 \%$ ethanol $+25 \%$ iso-amyl acetate, $50 \%$ ethanol $+50 \%$ isoamyl acetate, $25 \%$ ethanol $+75 \%$ iso-amyl acetate, and $100 \%$ iso-amyl acetate, $10-20 \mathrm{~min}$ each), critical point dried with $\mathrm{CO}_{2}$ in a Hitachi HCP-2 critical point drier (Tokyo, Japan), mounted on stubs, and coated with gold palladium in an SPI Module (Structure Probe Inc., West Chester, PA, USA) sputter coater. Occasionally, samples were captured obliquely. The samples were observed and micrographs taken with a Hitachi S-3000N SEM (Tokyo, Japan) operating at $30 \mathrm{kV}$. The tonal qualities of the images were adjusted, labels were added, and plates were assembled with Adobe Photoshop CS2 and CS3.

\section{Results}

\section{Habitat}

In our study, we found L. maackii plants growing as large deciduous shrubs about $5 \mathrm{~m}$ tall with erect woody stems (Fig. 1a). The stems are often multiple from the base of the shrub and up to $2-3 \mathrm{~cm}$ in diameter. The leaves are opposite, petiolate, and simple with entire ovate-lanceolate, acute to acuminate blades that are about $9 \mathrm{~cm}$ long, about $4 \mathrm{~cm}$ broad, and typically glabrous adaxially (Fig. 1b). Tomentose petioles are up to $6 \mathrm{~mm}$ long. The whole tree is filled with lovely red fruits during the fruiting period, which is typically from August to October (Fig. 1c).

Anatomy of abscission

\section{Prior to abscission}

No obvious cell division precedes abscission in $L$. maackii, and no abscission zone is evident in the petiole in early November. Three vascular bundles are embedded in the cortex (mesophyll) of the petiole and are arranged triangularly; the middle vascular bundle is larger than the other two (Fig. 2a). Each vascular bundle is comprised of adaxial xylem and abaxial phloem (Fig. 2b). In the petiole and leaf blade, the cortex is confluent with the adaxial and abaxial epidermis, the latter of which is decorated by many trichomes.

\section{Onset of abscission}

Upon the onset of the abscission process (mid November), a schism begins to form in the abaxial cortex about three to 

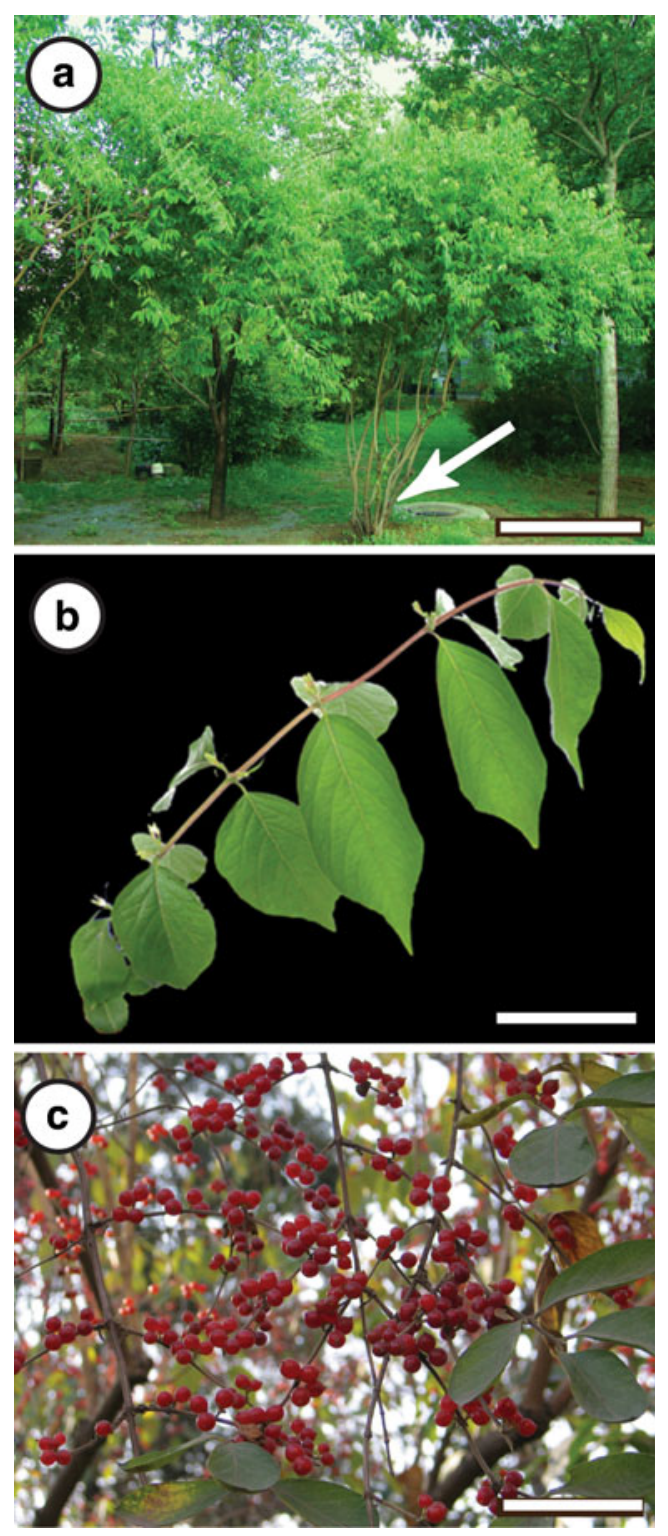

Fig. 1 Habitat and general morphology of Lonicera maackii plants. a Two shrubs growing at the Beijing Vocational College of Agriculture, photographed 1 August 2009 (arrows). The stems are multiple from the base of the shrub on the right. b Typical branch with opposite leaves, collected on 1 August 2009. c Tree with persistent fruit, observed 15 October 2009. Bar: a $2 \mathrm{~m}$, b $5 \mathrm{~cm}$, c $10 \mathrm{~cm}$

four cell layers in from the epidermis, creating an obvious gap as the interior cortical cells folds inward (Fig. 2c). The first cortical cells to separate are located distal to the petiole. Cortical cells on either side of the gap remain intact and enclosed in their cell walls, which suggests that the separation occurs at the middle lamella (Fig. 2d). Abscission occurs in the absence of cell divisions, and there is no obvious change in the cells of the petiole at this stage that would indicate the presence of an abscission zone. No similar gap is evident adaxially.

\section{Progression of abscission}

Throughout early December, the parenchyma cells of the abaxial cortex continue to detach at a region three to four layers in from the abaxial epidermis (Fig. 3a). The abaxial gap extends into the petiole and enlarges toward the vascular tissue so that the parenchyma cells of the phloem become disorganized. As a result, the petiole is thinner by about $300 \mu \mathrm{m}$. Also, at this time, a uniseriate layer of relatively enlarged cells (the abscission zone) becomes evident in the petiole.

\section{Completion of abscission}

By late December, leaf fall is imminent. At this time, the abaxial gap contacts the enlarged cells of the abscission zone, which are no longer intact, seemingly disrupted at their cell walls (Fig. 3b). The leaves undergo a slight yellowing and mottling just prior to leaf abscission. As the weight of the leaf is no longer supported by an intact petiole or blade, the vascular tissues undergo physical rupture (Fig. 3c). An adaxial gap never forms, and thus, when the leaf blade abscises at the end of December, the adaxial epidermis falls with the blade, while the abaxial epidermis and remnant outer three to four layers of cortical cells remains attached to the petiole in various degrees, depending on where physical rupture occurs (Fig. 3d).

\section{Discussion}

The leaf abscission process we observed in our SEM study of L. maackii does not fully conform to that documented in the better-studied model systems. In those species, an abscission zone is morphologically distinct prior to the onset of abscission (Roberts et al. 2000); no similar zone is evident in the L. maackii petiole until the process of abscission has already begun. Furthermore, typical abscission zones observed in very early stages of development are invariably described as tightly localized regions of relatively small cells lacking large vacuoles and any aspect of differentiation (Webster 1968; Addicott 1982). In contrast, the uniseriate layer that becomes prominent in the L. maackii petiole during abscission, which we have identified as the abscission zone, is comprised of highly vacuolated and relatively enlarged cells. Nonetheless, our observations are not unique: In I. sultani, cells comprising an abscission zone are not evident until abscission is underway (Gawadi and Avery 1950). Both L. maackii and I. sultani, while members of different orders, do belong to the asterid clade of the eudicots (APG II 2003).

We also note that the cortex of $L$. maackii leaves play a role in abscission, as it develops an "abaxial gap" at the 

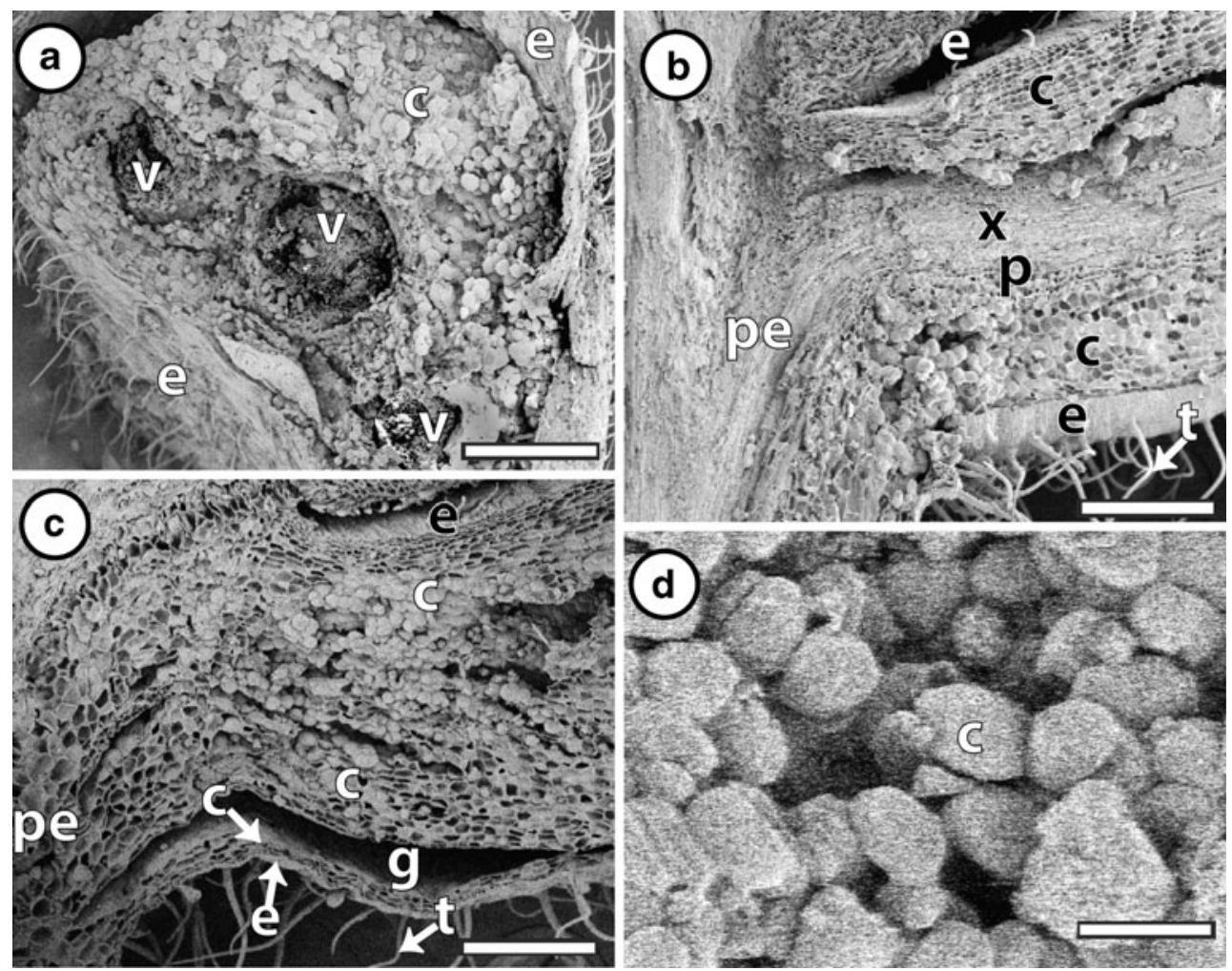

Fig. 2 Pre- and early events in leaf abscission. a Cross section of a petiole (pe) sampled 1 November 2009, prior to the onset of abscission. Three triangularly arranged vascular bundles $(v)$ are embedded in the cortex $(c)$ and surrounded by the epidermis $(e)$. b Longitudinal section through a leaf collected at the same time as a. Xylem $(x)$ is evident adaxially; phloem $(p)$, abaxially. The cortex is confluent with the adaxial and abaxial epidermis; the abaxial epidermis $(e)$ is oriented toward the bottom of each photograph. A

onset of abscission. Our observation was initially perplexing: At first, we thought perhaps there were problems with our tissue processing, but the consistent appearance of intact leaves prior to abscission in conjunction with the repeated observations of the abaxial gap during abscission in nearly all samples observed lead us to conclude that our micrographs represent the in vivo process. Again, in I. sultani, actual separation of cells begins in the hypodermis and extends into cortex and vascular bundles (Sexton 1976; Gawadi and Avery 1950). In that species, abscission is not thought to begin until the leaves are obviously senescent, and perhaps the situation is similar for L. maackii. Indeed, our observation that leaves became yellowed and mottled their abscission became imminent suggests that senescence is a prerequisite for leaf abscission in L. maackii.

With respect to the actual cell separation process in $L$. maackii, we observed three different mechanisms corresponding to three distinct regions: the abscission zone, the cortex (i.e., the abaxial gap), and the vasculature. The enlarged cells of the abscission zone seem to undergo a trichome $(t)$ is evident on the abaxial surface. c Onset of abscission, evident in a longitudinal section of a leaf sampled 15 November 2009. A gap $(g)$ in the cortex $(c)$ is forming near the abaxial epidermis $(e)$; the interior cortical cells fold inward (vascular tissue not captured in this section). d Oblique section that allows viewing of cortical cells $(c)$ on the inner surface of the gap. The cells are intact and enclosed in their cell walls. A trichome $(t)$ is evident. Bar: a $1 \mathrm{~mm}, \mathbf{b} 600 \mu \mathrm{m}$, c $500 \mu \mathrm{m}, \mathbf{d} 60 \mu \mathrm{m}$

programmed cell death or physical dissolution in which the cells lose integrity. In contrast, most reports for other plant species states that dissolution at the abscission zone occurs at the middle lamella only and that the separating cells remain intact (Facey 1950; Gawadi and Avery 1950; Webster 1968; Roberts et al. 2000). Even so, there are examples (e.g., Phaseolus vulgaris L.) in which both the middle lamella and the primary wall of cells in the abscission zone break down, after which the intact nucleus and cytoplasm are frequently extruded into the separation cavity (Webster 1968; Roberts et al. 2000); we think a similar process occurs in L. maackii.

The abaxial gap of L. maackii apparently arises by dissolution of the middle lamella, as the cortical cells remain intact; this observation corresponds to cell separation processes at the abscission zones of many other species and also to the cortical separation process of $I$. sultani (Sexton 1976; Gawadi and Avery 1950). Also, in agreement with the observations in many species, simple mechanical breakage severs the vascular tissue in $L$. maackii. 

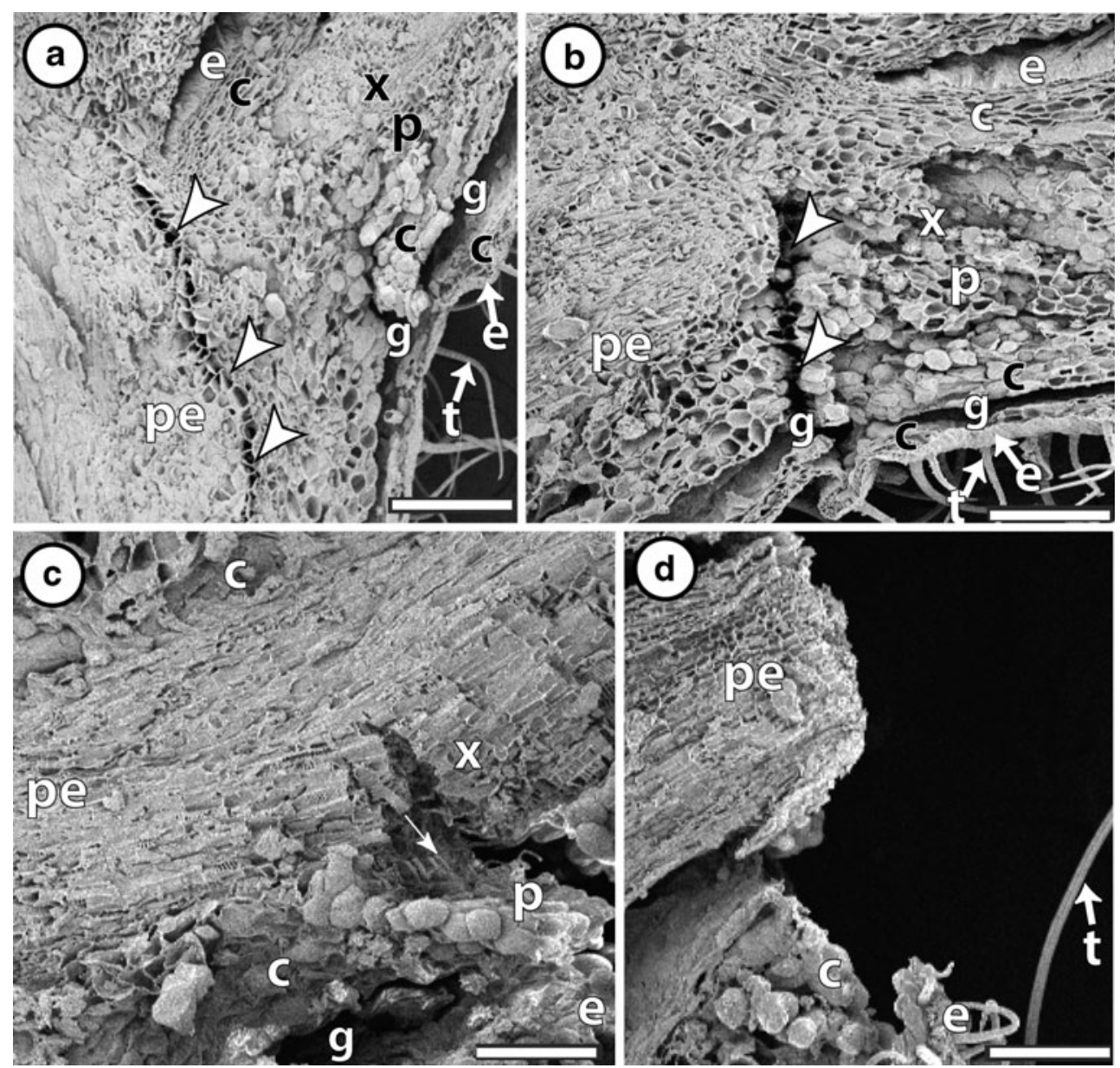

Fig. 3 Completion of leaf abscission. a Longitudinal section of a leaf sampled 1 December 2009. Parenchyma cells of the abaxial cortex (c) continue to detach at a region about three to four layers in from the abaxial epidermis $(e)$, and the gap $(g)$ enlarges toward the vascular tissue, disrupting the phloem $(p)$. A uniseriate layer of relatively enlarged cells (arrowheads) becomes evident in the petiole (pe). b Longitudinal section of a leaf from 15 December 2009. The abaxial gap $(g)$ in the cortex $(c)$ becomes confluent with the uniseriate layer of enlarged cells (arrowheads) in the petiole (pe); these cells no longer

Regardless of the genetic and developmental processes that govern abscission in L. maackii, the timing of abscission is probably also related to climate, especially day length and temperature; abscission in two related species of Lonicera, Lonicera sempervirens and Lonicera japonica, occurs earlier in the year in northern parts of its range, where the growing season is shorter due to a cooler climate and reduced day length (Leatherman 1955; Schierenbeck and Marshall 1993).

In conclusion, our simple but relevant SEM study has shown that leaf abscission in L. maackii begins with a separation of cortical cells at their middle lamella, followed by an enlargement and rupture of cells of the abscission zone in the petiole, and finally by mechanical rupture of the vascular tissues and remaining blade tissues. appear intact, as they are seemingly disrupted at their cell walls. $\mathbf{c}$ The vascular tissue from a leaf sampled at the same time as b showing physical rupture of the xylem $(x)$ and phloem $(p)$ near the petiole $(p e)$; remnants of vessel element walls are evident (small arrow), as is the abaxial gap $(g)$ in the cortex. d Remnant petiole (pe) after leaf abscission (30 December 2009). In this image, only a small component of the abaxial epidermis $(e)$ and outer three to four layers of cortical cells $(c)$ remain attached to the petiole. $t$ trichome. Bar: $\mathbf{a}, \mathbf{b}$ $600 \mu \mathrm{m} ; \mathbf{c}, \mathbf{d} 400 \mu \mathrm{m}$

This information is relevant for a popular ornamental like L. maackii, as such knowledge may be important in its possible future artificial culture. Furthermore, while the leaf abscission process we observed has similarities with model systems, aspects deviate from the expected, sometimes startlingly so. We suggest that leaf abscission needs further examination in more species, as what has been considered "typical" may not be fully representative.

Acknowledgments This work was supported by the fund for state key disciplines and the Chinese Academy of Sciences Laboratory [grant number: O5S0671204 provided to HFW] and a National Sciences and Engineering Research Council of Canada Discovery Grant [grant number 164375 provided to CRF]. We are very grateful to Zhi-xin Zhu, Jie Li, and Jian-fei Ye for experimental assistance, and we sincerely thank Xiao-min Ma for help with referencing. 
Conflict of interest The authors declare that they have no conflict of interest.

\section{References}

Addicott FT (1982) Abscission. University of California Press, London Angiosperm Phylogeny Group (APG) II (2003) An update of the Angiosperm Phylogeny Group classification for the orders and families of flowering plants: APG II. Bot J Lin Soc 141:399-436

Facey V (1950) Abscission of leaves in Fraxinus americana L. New Phytol 49:103-116

Gawadi AG, Avery GS Jr (1950) Leaf abscission and the so-called "abscission layer". Am J Bot 37:172-180

González-Carranza ZH, Lozoya-Gloria E, Roberts JA (1998) Recent developments in abscission: shedding light on the shedding process. Trends Plant Sci 3:10-14

Jensen WK (1962) Botanical histochemistry. WH Freeman, San Francisco Leatherman AD (1955) Ecological life-history of Lonicera japonica Thunb. Dissertation, University of Tennessee
Mackenzie KAD (1979) The structure of the fruit of the red raspberry (Rubus idaeus L.) in relation to abscission. Ann Bot 43:355-362

Roberts JA, Shindler BC, Tucker GA (1984) Ethylene-promoted tomato flower abscission and the possible involvement of an inhibitor. Planta 160:159-163

Roberts JA, Whitelaw CA, González-Carranza ZH, McManus M (2000) Cell separation processes in plants-models, mechanisms and manipulation. Ann Bot 86:223-235

Schierenbeck KA, Marshall JD (1993) Seasonal and diurnal patterns of photosynthetic gas exchange for Lonicera sempervirens and $L$. japonica (Caprifoliaceae). Am J Bot 80:1292-1299

Sexton R (1976) Some ultrastructural observations on the nature of foliar abscission in Impatiens sultani. Planta 128:49-58

Sexton R, Redshaw AJ (1981) The role of cell expansion in the abscission of Impatiens sultani leaves. Ann Bot 48:745-756

van Nocker S (2009) Development of the abscission zone. Stewart Posthar Rev 1:1-6

Webster BD (1968) Anatomical aspects of abscission. Plant Physiol 43:1512-1544

Xu B-S, Wang H-J (1988) Caprifoliaceae. In: Xu B-S (ed) Fl Reipub Pop Sin Science Press, Beijing 72:12-104 